\title{
Valutazione metabolica e gestione medica della nefrolitiasi: quale futuro?
}

\author{
M. Terribile' ${ }^{1}$ F. Forte ${ }^{2}$, C. Paglionico ${ }^{1}$ \\ ${ }^{1}$ Centro per lo Studio della Calcolosi Renale - www. calcolosirenale.it \\ Unità Operativa di Nefrologia e Dialisi e e Unità Operativa di Patologia Clinica, \\ "Ospedale dei Pellegrini", Napoli
}

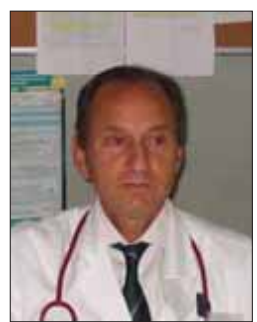

$\mathbf{N}$ egli ultimi venti anni vi è stata una progressiva riduzione di interesse nella valutazione metabolica e gestione medica della nefrolitiasi (NL).

Probabilmente l'introduzione della ESWL e delle metodiche endourologiche, facilitando notevolmente la eliminazione dei calcoli, hanno comportato un minore interesse verso l'approfondimento di tali tematiche.

Vi è stato però nel contempo un notevole miglioramento delle conoscenze della fisiopatologia della NL, con la diagnosi di specifiche anomalie e la messa in atto di altrettanto specifiche terapie.

È possibile infatti diagnosticare alterazioni di carattere metabolico o dietetico nutrizionale in più del $90 \%$ dei pazienti con calcolosi renale $(1,2)$.

Essendovi un'alta probabilità di recidiva di calcolosi dopo un primo episodio (3) ed essendo ancora più alta la probabilità di recidiva dopo un trattamento di ESWL (4) rimane necessaria una appropriata valutazione me- tabolica e gestione medica.

Una terapia medica selettiva si è dimostrata infatti in grado di ridurre la formazione di nuovi calcoli, diminuendo il ricorso a ripetute ESWL o a tecniche endourologiche che, pur essendo molto meno invasive rispetto alla chirurgia a cielo aperto, non sono prive di effetti collaterali.

E stato rilevato che con una adeguata terapia medica (5) si ottiene una riduzione dell' $85 \%$ della recidiva di calcolosi da calcio ossalato, che è la forma più comune di NL.

Inoltre, non raramente, la calcolosi renale è manifestazione di altra patologia (iperparatiroidismo, cistinuria, malassorbimento, rene a spugna midollare ecc) per cui un approfondito studio metabolico può non solo consentire di ridurre la frequenza di recidive, ma anche di scoprire e trattare la patologia primitiva.

Riteniamo però necessario che la valutazione metabolica sia di semplice esecuzione e non gravata da costi eccessivi per il paziente.

Inoltre, alla luce di una gestione in chiave "economica" della Unità Operativa, non deve comportare un aggra- vio di costi di gestione tale da rendere non conveniente l'attività di un Centro per la calcolosi renale.

Quali accertamenti deve effettuare un Centro per la calcolosi renale?

Vi è disaccordo in riferimento agli accertamenti per la valutazione diagnostica della NL.

In alcuni Centri si prevedono due protocolli di indagini, uno abbreviato per i pazienti che hanno prodotto un singolo calcolo, uno più estensivo per pazienti con NL ricorrente.

E stato però provato che pazienti produttori di un singolo calcolo possono avere la stessa incidenza e severità di disordini metabolici rispetto a pazienti con NL ricorrente (1). Uno studio metabolico abbreviato potrebbe essere insufficiente per i necessari chiarimenti diagnostici Pak et al (6) continuano ad effettuare il test da carico orale di calcio per discriminare tra le diverse forme di ipercalciuria nel caso di NL ricorrenti.

Nella maggior parte dei Centri per la NL tale test non viene però più utilizzato considerando la differenziazione 


\section{Giornale di Tecniche \\ Nefrologiche \\ \& Dialitiche \\ Anno XVI n ${ }^{\circ} 3$ \\ (c) Wichtig Editore 2004}

delle diverse forme di ipercalciuria di limitato significato pratico.

Il nostro approccio diagnostico, concorda, in linea di massima, con quelli più comunemente messi in atto dagli altri Centri. È semplice e non gravato da costi eccessivi.

Prevede dosaggi su urine delle $24 \mathrm{~h}$ (urea, creatinina, acido urico, $\mathrm{Na}, \mathrm{K}$, $\mathrm{Ca}, \mathrm{P}, \mathrm{Cl}, \mathrm{Mg}$, solfati, ammonio, cistina), su urine a digiuno (creatinina, $\mathrm{Ca}$ ), su siero (urea, creatinina, acido urico, $\mathrm{Na}, \mathrm{K}, \mathrm{Ca}, \mathrm{P}, \mathrm{Cl}, \mathrm{Mg}$ ) e la valutazione dello stato di saturazione urinario.

Sono poi talvolta effettuati esami di II livello in base al problema metabolico riscontrato.

Nelle ipercalciurie e/o ipercalcemie è effettuato dosaggio del iPTH.

Nelle ipercalciurie presumibilmente non assorbitive viene effettuata MOC più dosaggio di un marker di neoformazione (fosfatasi alcalina ossea) e di demolizione di tessuto osseo (desossipiridoline libere urinarie).

Gli esami sono effettuati mediamente a ritmo semestrale con possibili variazioni in base al diverso rischio litiasico.

Anche l'ecografia dei reni e delle vie urinarie è programmata a ritmo semestrale.

Qual è l'analisi costo-beneficio della profilassi medica della NL?

Uno studio italiano di Vitale et al (7) ha rilevato che la profilassi medica riduce del $62 \%$ la probabilità di recidiva e che ha un costo di $328 €$ per la prevenzione di ciascun episodio litogeno.

Parks e Coe (8) hanno valutato i costi di un programma di prevenzione della NL.

In base agli episodi di calcolosi prima e dopo l'istituzione della terapia medica hanno evidenziato un risparmio compreso tra 1 e 3 milioni \$ per 1000 pazienti/anno.

Robertson (9), effettuando una valutazione economica della profilassi medica della NL in Gran Bretagna per un periodo di trenta anni è pervenuto a risultati similari.

In questi ultimi due studi non è stato considerato la perdita di giornate di lavoro che rappresenta un ulteriore costo a favore della prevenzione medica.

Pertanto il relativo basso costo di una valutazione metabolica e gestione medica potrebbe giustificarne una sua più ampia applicazione, limitando il ricorso a tecniche urologiche sicuramente gravate da costi maggiori.

Qual è l'onere economico per il paziente?

Per gli esami laboratoristici di routine il paziente paga un ticket di $24 €$, il $38.7 \%$ del costo totale $(62.27 €)$.

Il costo della visita ambulatoriale per NL è di 18.60 euro.

Il costo della ecografia reni e vie urinarie prevede un ticket di 36.15 euro.

Gli esami di II livello più comunemente effettuati presentano i seguenti costi: MOC $28 €$, fosfatasi alcalina ossea e desossipiridoline urinarie 36 euro.

$\grave{E}$ preferibile il laboratorio specialistico della Unità Operativa di nefrologia o il laboratorio centralizzato?

Solitamente ogni Centro per la calcolosi renale ha un suo specifico laboratorio dove effettuare le indagini di carattere non routinario. Ciò presenta alcuni vantaggi quali la maggiore facilità a ottenere controlli di qualità e a impostare nuove procedure diagnostiche specifiche per la NL.

Gli svantaggi sono rappresentati dal consumo di risorse economiche quasi sempre eccessivo. Nel nostro caso abbiamo ritenuto preferibile accentrare le procedure diagnostiche presso il laboratorio centralizzato, ma con uno stretto rapporto di collaborazione con personale laureato ampiamente motivato che si dedica specificamente ai dosaggi del nostro Centro, ottenendo risultati ampiamente soddisfacenti.

\section{È possibile la gestione integrata} (nefrologo-urologo) della NL?

Sarebbe quanto mai auspicabile una gestione integrata della NL.

Purtroppo, in base alla nostra espe- rienza, nella pratica quotidiana è poche volte possibile. L'urologo non raramente interpreta l'intervento del nefrologo nella NL come una intrusione in un settore di suo esclusivo interesse e ha, conseguentemente, un atteggiamento di carattere ostativo. Probabilmente saranno differenti le esperienze di quei Centri per la calcolosi presenti sul territorio nazionale da molti anni, dal periodo pre-ESWL, in cui era scontata e anzi da tutti auspicata una valutazione metabolica e gestione medica della NL; tali centri probabilmente non hanno incontrato tali problemi.

Al Centro per lo Studio della calcolosi renale sono indirizzati pazienti specialmente dai medici di famiglia e dai nefrologi. Puramente occasionali sono i pazienti inviati dagli urologi che pur sono in gran parte a conoscenza delle nostre finalità e dell'attività svolta, che sono di tipo complementare e non conflittuale rispetto a quella dell'urologo.

$\grave{E}$ opportuno che il nefrologo intervenga nella scelta delle procedure di trattamento dei calcoli?

Al nefrologo che gestisce un ambulatorio di calcolosi renale viene chiesto molto frequentemente quale possa essere il trattamento ottimale (ESWL, ureteroscopia o PCNL). Sono trattamenti di stretta competenza urologica; tuttavia non si può semplicisticamente non rispondere al quesito posto dai pazienti. Riteniamo che l'atteggiamento più corretto sia quello di citare le linee guida europee e/o statunitensi circa la terapia di elezione facendo nel contempo presente la possibile diversa valutazione dell'urologo. In base alla nostra esperienza vi è un eccessivo utilizzo della ESWL da parte dell'urologo anche in quelle condizioni cliniche in cui è improbabile che sia coronata da successo (calcolosi caliceale inferiore, calcolosi a stampo).

Quale futuro per la valutazione metabolica e gestione medica della NL?

Riteniamo che vi sia un futuro per la valutazione metabolica e gestione 
medica della NL.

La nostra esperienza con il Centro per lo Studio della calcolosi renale lo dimostra.

Nato nel 2002, ad esso fanno riferimento circa 300 pazienti calcolotici. Il Centro presenta una continua espansione.

La ragione di tale successo è probabilmente ascrivibile al fatto che a) la maggior parte dei pazienti con NL è interessata nel conoscere perché produce i calcoli; b) è disposta a sottoporsi a terapia dietetica e/o farmacologica per evitare il rischio di recidive; c) gli accertamenti diagnostici praticati sono relativamente semplici e non onerosi.

\section{BIBLIOGRAFIA}

1. Preminger G. Medical evaluation and treatment of nephrolithiasis. Semin Urol 1994, 1: 51-64.

2. Pak CY. Physiological basis for absorbitive and renal hypercalciurias. Am J Physiol 1979; 237: 415-23.

3. Sutherland J, Parks J, Coe F. Recurrence after a single renal stone in a community practice. Miner Electrolyte Metab 1985; 11: 267.

4. Sun BY, Lee YH, Jiaan BP. Recurrence rate and risk factors for urinary calculi after ESWL. J Urol 1996; 156: 903-6.

5. Pak CY. Is selective therapy of recurrent nephrolithiasis possible? Am J Med 1981; 71: 615-22.

6. Pak CY, Kaplan RA, Bone H. A simple test for the diagnosis of absorptive, resorptive and renal hypercalciuria. N Engl J Med 1975; 292: 497-500.

7. Vitale C, Marangella M, Bagnis C. Effetti a lungo termine della terapia medica nel decorso clinico della nefrolitiasi. Giorn It Nefrol 2001; 5: 524-9.

8. Parks JH, Coe FL. The financial effects of kidney stone prevention. Kidney Int 1996; 50: 1706-12.

9. Robertson WG. Medical management of urinary stone disease. EBU Update Series 1998; 7: 139-44. 